\title{
Supporting Information: Frugal Science powered by curiosity
}

Gaurav Byagathvalli ${ }^{1}+$, Elio J. Challita ${ }^{2} \ddagger$, and M. Saad Bhamla ${ }^{*}$

1. School of Chemical and Biomolecular Engineering, Georgia Institute of Technology, Atlanta, GA 30332

2. Mechanical Engineering, Georgia Institute of Technology, Atlanta, GA, 30311, USA

\begin{tabular}{llll} 
Technology Name & Frugal Version & Commercial Version & References \\
\hline ElectroPen/ePatch & $\$ 0.23-1.00$ & $>\$ 2,000$ & $1-2$ \\
Paperfuge & $\$ 0.20-1.00$ & $>\$ 700$ & $3-4$ \\
$\begin{array}{l}\text { Paper-based } \\
\text { diagnostics }\end{array}$ & $\$ 0.01-0.50$ & $>\$ 10$ & $5-7$ \\
Foldscope & $\$ 0.58-2.00$ & $>\$ 500$ & 8 \\
Triboelectric X-ray & $\$ 5.00-10.00$ & $>\$ 125,000$ & $9-10$ \\
Bubble Wrap Assay & $\$ 0.01-0.20$ & $\$ 0.60$ & 11
\end{tabular}

Table S1. Summary of cost comparisons between frugal science tools and comparable commercial devices.

References

1. Byagathvalli, G., Sinha, S., Zhang, Y., Styczynski, M. P., Standeven, J., \& Bhamla, M. S. (2020). ElectroPen: An ultra-low-cost, electricity-free, portable electroporator. PLoS biology, 18(1), e3000589.

2. Xia et al. (2021) An ultralow-cost electroporator with microneedle electrodes (ePatch) for SARS-CoV-2 vaccination.

3. Bhamla, M. S., Benson, B., Chai, C., Katsikis, G., Johri, A., \& Prakash, M. (2017). Handpowered ultralow-cost paper centrifuge. Nature Biomedical Engineering, 1(1), 1-7.

4. Byagathvalli, G., Pomerantz, A., Sinha, S., Standeven, J., \& Bhamla, M. S. (2019). A 3Dprinted hand-powered centrifuge for molecular biology. PLoS biology, 17(5), e3000251.

5. King, R. (2020, June 10). Study: Annual costs for diagnostic tests up to $\$ 25 B$, antibody tests up to $\$ 19 B$. FierceHealthcare. Retrieved September 28, 2021, from https://www.fiercehealthcare.com/payer/study-annual-costs-for-diagnostic-tests-up-to25b-and-antibody-tests-up-to-19b.

6. Outpatient Lab, Diagnostic and other ancillary charges for healthcare services. Lee Health. (n.d.). Retrieved September 28, 2021, from https://www.leehealth.org/billing/estimates-costs/how-much-do-procedurescost/outpatient-lab-diagnostic-and-other-ancillary-charges-for-healthcare-services.

7. Martinez, A. W., Phillips, S. T., Whitesides, G. M., \& Carrilho, E. (2010). Diagnostics for the developing world: microfluidic paper-based analytical devices. 
8. Cybulski, J. S., Clements, J., \& Prakash, M. (2014). Foldscope: origami-based paper microscope. PloS one, $9(6)$, e98781.

9. Lori Webb \& Webb, L. (n.d.). Purchasing insight: Portable X-ray. Healthcare Finance News. Retrieved September 28, 2021, from

https://www.healthcarefinancenews.com/blog/purchasing-insight-portable-xray\#: :text=Hospitals\%20with\%20an\%20xisting\%20analog,and $\% 20$ type $\% 20$ of $\% 20$ det ector $\% 20$ selected.

10. Hird, J. R., Camara, C. G., \& Putterman, S. J. (2011). A triboelectric x-ray source. Applied Physics Letters, 98(13), 133501.

11. Bwambok, D. K., Christodouleas, D. C., Morin, S. A., Lange, H., Phillips, S. T., \& Whitesides, G. M. (2014). Adaptive use of bubble wrap for storing liquid samples and performing analytical assays. Analytical chemistry, 86(15), 7478-7485. 